\title{
CUATRO CARTAS DE SIR ISAAC NEWTON AL DOCTOR BENTLEY QUE CONTIENEN ALGUNOS ARGUMENTOS EN FAVOR DE LA EXISTENCIA DE UNA DEIDAD. (1692-1693)
}

\section{Introducción}

Las cartas que presentamos a continuación fueron escritas por Newton entre los años 1692 y 1693, es decir, con posterioridad a la primera edición de los Principia (1686), y poco antes de su "crisis mental" de este último año; aunque sólo serían publicadas por los ejecutores testamentarios de Richard Bentley en 1756, esto es, 29 años después de la muerte del gran físico, astrónomo y matemático inglés.

De acuerdo con el influyente historiador de la ciencia Alexandre Koyré, estas "cuatro cartas [...] constituyen uno de los más preciosos e importantes documentos para el estudio e interpretación del pensamiento newtoniano" (Newtonian Studies, The University of Chicago Press, 1968, p. 202). Sin embargo, el significado de estas cartas no será en absoluto evidente para el lector contemporáneo que no esté familiarizado con el contexto histórico, científico y religioso en el que se escribieron.

Como ya lo hemos argumentado en otra parte, ${ }^{1}$ la ciencia moderna apareció en la historia de Occidente como una nueva concepción del mundo y del conocimiento, que surgió a partir del propio horizonte del pensamiento medieval, del que se fue disociando muy lenta y trabajosamente. De allí que no tenga nada de sorprendente que el pensamiento científico de Newton esté profundamente influido por conceptos, problemas y planteamientos teológicos, religiosos y metafísicos, propios de la matriz medieval en la que se gestó. Lo que caracteriza precisamente la obra de las principales figuras del renacimiento científico (Kepler, Galileo, Bacon, Descartes), es el intento de delimitar una esfera de investigación separada y libre de la intromisión de la religión y la teología. Esta exigencia de distinguir las cuestiones teológicas de las propiamente científicas, a menudo terminó por repudiar la búsqueda

1 H.H. Benítez, "El caso Galileo y las raíces del conflicto entre religión y ciencia en la época moderna", Revista Mapocho, Biblioteca Nacional, Santiago de Chile, no. 38, segundo semestre 1995, pp. 173-198. 
de las causas finales, por encontrarse más allá de su campo propio. Así, por ejemplo, lo manifiesta Francis Bacon en su Novum organum, al declarar que "La investigación de la causa final está tan lejos de ser provechosa a las ciencias que más bien las corrompe si no es [en el estudio] de las acciones humanas" (trad. de R. Frondizi, Losada, Buenos Aires, 1949, p. 172).

Por su parte Descartes, en los Principios de la filosofía, expresa, cautelosa pero inequívocamente, la misma posición separatista en los siguientes términos:

\begin{abstract}
Atendiendo al inmenso poder de Dios, no podemos estimar que haya hecho jamás algo que no fuera absolutamente perfecto. Pero, con todo, para comprender la naturaleza de las plantas o de los hombres, es mucho mejor considerar cómo poco a poco pueden nacer de simiente, que no cómo hayan sido creadas por Dios en el origen primero del mundo. (Trad. de Halperin, Losada, Buenos Aires, 1941, parte tercera, párrafo XLV)
\end{abstract}

Pero la postulación de esta "separación de esferas" no debe entenderse como si hubiera implicado propósitos de completa ruptura con la cosmovisión medieval. Por el contrario, tanto Galileo en Italia, como Descartes y Gassendi en Francia, se propusieron no un rompimiento, sino una suerte de entendimiento o compromiso entre la ciencia naciente y la tradición y tutela intelectual de la Iglesia católica. Por desgracia, en aquellos países tal entendimiento no llegó a cristalizar, y lo que pudo haber sido una relación armónica entre la ciencia y la religión se transformó, ya en 1616 (fecha de la prohibición del copernicanismo por la Inquisición romana), en un conflicto abierto.

Tomando como caso paradigmático la astronomía, la posición de la Iglesia frente a las cuestiones científicas antes del cisma protestante era bastante laxa, y no exigía de sus fieles la creencia obligatoria en ninguna doctrina específica en materias astronómicas o cosmológicas. Incluso durante el siglo xv el cardenal Nicolás de Cusa pudo defender, de palabra y por escrito, una cosmología infinitista de origen neoplatónico, sin ser criticado ni perseguido por la Iglesia, a pesar de que ella se encontraba en manifiesto desacuerdo con las Escrituras. Sin embargo, con el advenimiento de la Reforma y la serie de profundos conflictos religiosos y políticos que traería consigo, la situación cambió radicalmente. A partir del Concilio de Trento, que representó una reafirmación del aristotelismo en lo científico, y del literalismo bíblico en lo religioso, la actitud de la Iglesia se tornará más conservadora y adoptará una conducta represiva hacia cualquier manifestación, supuesta o real, de disidencia intelectual. El impulso que los nuevos descubrimientos y teorías astronómicas le imprimen a la ciencia emergente comienza a acentuar el temor de las iglesias, católica y protestante, hacia los efectos que aquéllos 
pudieran ejercer sobre su hegemonía intelectual y política en crisis. El "secularismo" de la ciencia, con su énfasis sobre las causas naturales y la razón, la hará sospechosa frente a una sociedad en la que la religión ejercía una enorme influencia intelectual y política.

En la Inglaterra de Newton, sin embargo, el desarrollo de la ciencia no conducirá a un choque con la religión, como el que se dio en los países sometidos al control intelectual de la Contrarreforma. La explicación de esto parece encontrarse tanto en la propia actitud de la Iglesia protestante hacia la ciencia en el siglo xvir, como en el hecho de que los científicos ingleses se las arreglaron para conciliar la filosofía de la naturaleza subyacente en la ciencia triunfante, es decir, el mecanicismo o filosofía corpuscular, con los principios de la teología y la religión cristianas dominantes en la sociedad del periodo de la Restauración. Esto exigió desde el primer momento que los científicos, influidos por el ejemplo de Pierre Gassendi, buscaran "expurgar" la teoría corpuscular, de sospechoso origen pagano y ateo, de aquellos elementos que pudieran interpretarse como en conflicto con el teísmo y el espiritualismo cristianos. ${ }^{2}$ De allí la necesidad de denunciar, por un lado, el materialismo y ateísmo epicúreo o hobbesiano, y por la otra, de demostrar la compatibilidad y complementariedad entre los propósitos cognoscitivos de la ciencia y los de la religión, en los momentos en que se producía en Inglaterra una fuerte insurgencia atea. ${ }^{3}$

Estos hechos aportan algunos de los antecedentes a partir de los cuales pueden explicarse los esfuerzos de Robert Boyle, Richard Bentley, Samuel Clarke y de tantos otros científicos teólogos ingleses, por combatir públicamente el ateísmo y el materialismo con los argumentos de la teología natural anglicana. Las lecciones-sermones que suministraron la oportunidad para la redacción e intercambio de las cartas que aquí introducimos se inscriben claramente en el contexto referido; su origen inmediato es la muerte del gran químico, físico, teólogo y filósofo mecanicista Robert Boyle, quien al fallecer el 30 de diciembre de 1691, dejó expresamente establecido en su

2 Curiosamente, la influencia que esta suerte de "atomismo cristianizado" postulado por Gassendi ejerció sobre los científicos franceses (quienes en su mayoría aceptaban el mecanicismo cartesiano), fue mucho menor que la que tendría en Gran Bretaña.

3 "la evidencia indica que a fines del siglo xvi el ateísmo en Inglaterra fue más frecuente que a mediados del siglo xviI. Hubo una explosión de ateísmo, particularmente en el periodo de la Restauración, en su mayor parte confinada a las clases altas, y basada fundamentalmente en el pensamiento de Hobbes" (David Berman, A History of Atheism in Britain. From Hobbes to Russell, Routledge, Londres, 1990, p. 48). Un excelente análisis del carácter de la reacción antiatea entre los científicos en aquel periodo lo ofrece Michael Hunter en su artículo: "Science and Heteronomy", que forma parte del volumen titulado: Reappraisals of the Scientific Revolution, David C. Lindberg y Robert S. Westman (comps.), Cambridge University Press, Cambridge, 1991, pp. 437-460. 
testamento que debía destinarse la suma de cincuenta libras anuales de aquel entonces al pago de un teólogo o cura predicador de Londres, para que dictara cada año ocho sermones en una parroquia local que "probaran la religión cristiana contra infieles bien conocidos, es decir, ateos, deístas, paganos, judíos y mahometanos, aunque sin rebajarse a las controversias entre cristianos" (Louis Trenchard More, The Life and Works of the Honorable Robert Boyle, Oxford University Press, Londres, 1944, p. 132).

Recaería sobre el reverendo Richard Bentley (1662-1742), Master del Trinity College, Universidad de Cambridge, y posteriormente Doctor en Teología, quien entonces tenía sólo 30 años de edad, la responsabilidad de hacerse cargo de los ocho primeros sermones, cuyos títulos expresan su estrecho apego a los deseos de Boyle: 1. "La locura del ateísmo y el deísmo incluso con respecto a la vida presente"; 2 . "La materia y su movimiento no pueden pensar"; 3,4 y 5. "Refutación del ateísmo a partir de la estructura del cuerpo humano"; 6, 7 y 8 . "Una refutación del ateísmo a partir del origen y estructura del mundo".

Es en la preparación de estos últimos sermones que Bentley, quien se había propuesto emplear la ciencia newtoniana con fines apologéticos, pero que no sabía suficientes matemáticas como para entender las complejas formulaciones de los Principia, se verá obligado a solicitar la ayuda de Newton. Por desgracia para los estudiosos, a excepción de una (la tercera), no se han encontrado las cartas que Bentley escribiera a Newton, de modo que el contenido de éstas sólo puede reconstruirse aproximadamente a partir de las respuestas dadas por el gran físico y matemático. Con la ayuda del siempre informativo Koyré, las principales preguntas que Bentley le planteó a Newton habrían sido las siguientes:

1. Si acaso el Sistema Solar pudo haberse formado (a partir de una distribución uniforme de la materia en el espacio), como efecto de la acción de puras causas naturales; y si una vez que Dios creó los planetas, los movimientos de éstos pudieron haberse originado en la acción de la pura gravitación.

2. Al crear Dios los planetas a cierta distancia del Sol, ¿no habrá podido darles sus movimientos orbitales mediante puras causas naturales?; es decir, imprimirles tanto un movimiento gravitacional hacia el Sol, como un impulso transversal calculado para hacerlos rotar en torno a éste.

3. ¿Pudieron los planetas haber adquirido progresivamente sus velocidades respectivas por la sola acción de sus pesos, en vez de haberlas adquirido de modo instántaneo por la acción de Dios? 
4. Si se supone que se formaron en alguna región distante y más alta que el Sol, ¿cómo pudieron los planetas adquirir sus movimientos transversales sin un poder divino que se los imprimiera? ( $C f r$. Koyré, op. cit., pp. 203-205.)

A nuestro juicio la clave para la comprensión del significado de las respuestas que dio Newton a cada una de estas preguntas se encuentra en su posición respecto de las relaciones entre ciencia y religión. En cuanto a esta cuestión, como lo ha destacado el historiador de las ideas Frank Manuel, Newton adoptó una posición que denomina "separatista", muy semejante a la postulada antes por Galileo y Descartes; y, como ellos; fue partidario de la doctrina de "los dos libros", el de las Escrituras y el de la Naturaleza, que aunque complementarios son tan claramente diferentes que deben mantenerse separados. De allí que Newton afirmara inicialmente que "religión y filosofía [natural] deben preservarse distintas. No debemos introducir revelaciones divinas en la filosofía [natural] ni opiniones filosóficas [es decir, científicas] en la religión" (Isaac Newton, "Seven Statements on Religion", Theological Manuscripts, H. McLachlan (comp.), Liverpool, 1950, p. 58).

Sin embargo, Newton nunca fue consistente con dicha posición y en el interior mismo de su obra científica irán adquiriendo una importancia cada vez mayor las formulaciones y conceptos metafísicos recibidos de su teísmo anglicano y de las influencias de Isaac Barrow, Henry More y otros "platónicos de Cambridge", hasta el extremo de que en el Scholium general a la segunda edición de los Principia (1713) el gran físico y matemático parece haber abandonado completamente su separatismo inicial, al declarar que:

si Dios redujo a orden el sistema del Sol y los planetas, las causas finales tendrán un lugar en la filosofía natural, y será legítimo investigar con qué fin fue fundado el mundo, con qué fin fueron formadas las extremidades de los animales, y por medio de qué sabiduría tienen ellas un orden tan elegante. (Cito aquí de una de la redacciones originales del Scholium reproducidas por Rupert Hall y Marie Boas en su edición de los Unpublished Scientific Papers of Isaac Newton, Cambridge University Press, 1962, p. 360)

Por cierto, Newton, como la totalidad de los científicos ingleses de su época, era creyente y veía en la ciencia un camino de acceso indirecto a Dios, complementario con la revelación, de modo que para él ciencia y religión no aparecían en competencia o conflicto, sino en completa armonía. Pero Newton no hubiera sido el científico que fue si no hubiera adoptado, simultáneamente, en algún grado, la creencia de que la religión y la metafísica no pueden inmiscuirse directamente en las explicaciones científicas. 
El estudio circunstanciado de la naturaleza exige una suerte de suspensión momentánea de la creencia en Dios, por lo menos en lo que se refiere a las causas inmediatas de los fenómenos, de lo contrario Dios se transforma en lo que Spinoza llama el "asilo de la ignorancia", es decir, en un recurso prematuro a la explicación metafísica allí donde se desconocen las causas físicas. Como lo señala tan acertadamente Robert Boyle en un pasaje clave citado por Burtt:

Porque para explicar un fenómeno no es suficiente asignarlo a una [causa] eficiente general, sino que debemos mostrar inteligiblemente la manera particular como aquella causa general produce el efecto propuesto. Un investigador tendría que ser muy torpe si, al demandar una explicación del fenómeno del reloj, se contentara con que se le dijera que es un motor hecho por un relojero; aunque no se le explicara nada acerca de la estructura y funcionamiento conjunto del resorte, ruedas, áncora y otras partes del motor, y de la manera como ellas interactúan y cooperan para hacer que los punteros marquen las verdaderas horas del día. (E.A. Burtt, Metaphysical Foundations of Modern Science, Doubleday, Nueva York, 1954, p. 178)

Es en este contexto donde las cartas que presentamos aquí cobran su verdadero significado, revelando un Newton para el que prácticamente ha desaparecido el límite entre ciencia y religión, al producirse en su pensamiento maduro una verdadera fusión de los intereses científicos y religiosos. Como puede verse, hay una gran diferencia entre los propósitos, comunes a todos los científicos-creyentes, de "complementar" la ciencia con la religión (o más bien la religión con la ciencia), y proponerse una fusión de ambas. La complementación no es contradictoria con la separación de esferas, más bien la presupone; mientras que la fusión implica, precisamente, la negación de la separación entre ciencia y religión. La compleja, y en última instancia inconsistente, posición de Newton ante esta cuestión ha confundido a más de un estudioso de su obra. Por ejemplo, Amos Funkestein, en su libro Theology and the Scientific Imagination, from the Middle Ages to the Seventeenth Century (Princeton University Press, 1986) sostiene que en el siglo xvi, en vez de una separación entre ciencia y religión, lo que en realidad se habría producido fue una fusión sin precedentes de éstas, y que Newton constituiría el ejemplo por excelencia de este procedimiento. Tal interpretación es, sin embargo, difícilmente conciliable con las explícitas declaraciones separatistas, no sólo de Newton, sino de la totalidad de los héroes de la Revolución Científica, y toma, equivocadamente, la postura tardía del gran físico, astrónomo y matemático inglés por su única posición acerca de las relaciones entre la ciencia y la religión. 
Aunque no ofreceremos aquí un comentario detallado de estas cartas, ${ }^{4}$ queremos al menos destacar brevemente algunos de sus puntos más importantes. A lo largo de ellas Newton emplea una serie de argumentos supuestamente probatorios de una intervención divina inmediata en la formación de nuestro Sistema Solar, pero todos ellos pueden reducirse a la idea de que su complejidad, organización, armonía y estabilidad no podrían ser el resultado de puras causas naturales ciegas, tal como lo habrían postulado los atomistas griegos. Aquí se expresa a todas luces el apego de Newton a los argumentos de la teología natural anglicana, con su radical subestimación del poder autoformativo y organizador de la ciega materia inanimada. Pero se muestra también alli el radical "fijismo" de su concepción cosmológica. Pues al concebir la formación del Sistema Solar como un acontecimiento único que habría tenido lugar en el momento mismo de la creación, y no como un proceso evolutivo a través del tiempo (tal como lo planteaba la hipótesis cartesiana de la formación del sistema planetario que él rechaza categóricamente desde su primera carta), no le quedaba, obviamente, a Newton otra salida que echar mano del recurso precipitado a la intervención de una deidad. La cuestión que se pone aquí de manifiesto, entonces, es la inconsistencia que se encontraba en la base del intento por parte del físico y matemático inglés de resolver una cuestión cosmológica, es decir, científica, por medio de una hipótesis metafísica: la voluntad y propósitos de Dios. Esto lo entendió muy bien el joven Kant, quien, en su Allgemeine Natureschichte und Theorie des Himmels, de 1755, se encargó de refutar la posición expresada por Newton en las cartas a Bentley, y según la cual el orden y la armonía de nuestro sistema solar habrían sido el efecto de la intervención inmediata de Dios, al demostrá que pueden explicarse como efecto de la materia abandonada a sus propias leyes mecánicas de movimiento. Lo importante aquí es entender que aunque tanto Newton como Kant eran creyentes de Dios, sólo Newton (violando sus propios cánones de razonamiento científico) traspasa ilícitamente la frontera que separa a la ciencia de la religión y la teología, mientras que Kant, aunque cree en que la explicación metafísica última de las propiedades de la materia que dieron origen al Sistema Solar se encontraría en la voluntad divina, en su hipótesis de la formación del sistema planetario se mantiene estrictamente dentro de los límites de la explicación naturalista. (Véase: Immanuel Kant, Historia general de la naturaleza y teoría del cielo, trad. Jorge E. Lunqt, Juárez Editor, Buenos Aires, 1969.)

4 El mejor examen detallado de las Cartas a Bentley lo ha hecho Paolo Casini en el capítulo segundo de su obra El universo máquina. Orígenes de la filosofía newtoniana, Ediciones Martínez Roca, Barcelona, 1971, pp. 61-90. 
El pasaje final de la primera carta, donde Newton rechaza la afirmación de Bentley de que el origen de la inclinación del eje de la Tierra pudiera ser efecto de la voluntad y presciencia divinas, muestra cómo el "Argumento del Diseño" puede constituirse en una especie de Deus ex Machina que se emplea para dar cuenta de cualquier hecho natural cuya causa precisa se desconoce. Newton se muestra aquí algo más cauto que el reverendo, pero es evidente que el recurso a Dios como explicación última está siempre expuesto al riesgo de que el desarrollo ulterior del conocimiento científico lo muestre como precipitado.

La referencia a la compleja cuestión de los infinitos en la segunda carta corresponde a la respuesta de Newton a la hipótesis, expresada por Bentley, "de que las masas, atraídas por todas partes con fuerzas infinitas, se mantendrán en equilibrio 'porque todos los infinitos son iguales'" (Paolo Casini, El universo máquina, p. 81). La réplica del gran físico, astrónomo y matemático fue categórica: la conclusión cosmológica extraída por Bentley es falsa porque está basada en una suposición igualmente falsa, propia de la concepción vulgar del infinito, y según la cual un infinito sería equivalente a cualquier otro.

A partir de los antecedentes proporcionados antes se entiende por qué en la tercera carta Newton es tan cortante en.su advertencia a Bentley en el sentido de que no le atribuya a él la idea de que la gravedad sería inherente a la materia, pues su posición teísta lo hacía rechazar cualquier posible asociación con la concepción epicúrea (que postulaba la gravedad como una propiedad esencial de los átomos), es decir, rechazaba ser identificado con aquellos que consideraba sus enemigos declarados: los materialistas y ateos de formación epicúrea, cartesiana o hobbesiana.

En la misma carta encontramos la conclusión de que "la gravedad puede ser causada por un agente que actúa constantemente de acuerdo con ciertas leyes; pero determinar si este agente es material o inmaterial lo he dejado a la consideración de los lectores". Esta afirmación ha hecho creer a algunos historiadores de la ciencia moderna (Casini entre otros) que Newton sostenía una posición no categórica acerca de este punto, pero en realidad esto no era así, tal como lo revela el contenido del propio Scholium general a los Principia, donde aquel "agente" aparece descrito como si tuviera propiedades inequivocamente espirituales. Esto no es una cuestión menor, porque como lo han demostrado los estudios de Burtt, Cohen y Hall, Dios cumple un papel determinante en la física y la cosmología newtonianas, lo que se muestra no sólo en su concepción del tiempo y el espacio absolutos, sino también de la gravitación y del "Sensorium".

La presente traducción se hizo a partir de la versión facsimilar de las Cartas a Bentley contenida entre las páginas 279 y 312 de los Isaac Newton's 
Papers \& Letters on Natural Philosophy, Harvard University Press, Cambridge Massachusetts, 1978, editados y con una introducción del emérito estudioso norteamericano de la obra de Newton, I. Bernard Cohen. A contrapelo con la manera tradicional de traducir a nuestra lengua, que hace prácticamente imposible el estudio filosófico de un texto, he tratado de suministrar al lector una traducción lo más ajustada y literal posible, buscando reproducir, no sólo la precisión y economía, sino incluso las peculiaridades sintácticas del inglés del siglo xvıı utilizado por Newton. La única excepción a este literalismo ha sido la eliminación del uso de la mayúscula en la primera letra de los sustantivos, tan común en las obras impresas en aquella época.

Por lo que sabemos, estas cartas no han sido hasta ahora vertidas en su totalidad a nuestra lengua. Existe, eso sí, una traducción parcial al español de la primera carta a Bentley, que forma parte de la excelente colección de textos científicos escogidos y traducidos por Roberto Torretti, publicada con el título general de Filosofia de la naturaleza, Editorial Universitaria, Santiago de Chile, 1971, pp. 161-164.

Con la presente traducción nos hemos propuesto poner al alcance de los lectores de Diánoia un documento de gran importancia en la historia de la revolución intelectual que dio origen a la ciencia moderna. Además, nos parece que los planteamientos contenidos en estas cartas permiten visualizar parte del enorme influjo que sobre las ideas cientificas han ejercido, a lo largo de su historia, los compromisos metafísicos y religiosos de sus creadores; así como también tener una idea de la tensión entre éstos y los impulsos puramente naturalistas de conocimiento que se dieron en la mente de aquellos que, además de ser grandes hombres de ciencia, eran también en su mayoría individuos profundamente religiosos. Aunado a lo anterior, estas cartas permiten ilustrar el doble carácter de la "teoría de la diferenciación de esferas", de la que hablamos al comienzo. Es decir, en primer lugar, su aspecto histórico, que explica el origen de la ciencia moderna a partir de una progresiva separación y diferenciación de su territorio propio y del perteneciente a la religión, la metafísica y la teología. El segundo aspecto, de carácter epistemológico, nos muestra la necesidad de reconocer e identificar esta separación como constitutiva de la ciencia misma, y de mantenerla y respetarla.

Las respuestas de Newton a las preguntas de Bentley muestran muy bien cómo el intento de mezclar y poner en el mismo nivel del discurso las explicaciones naturalistas y las explicaciones metafísico-religiosas redunda siempre en detrimento de la ciencia. Pues es indudable que la investigación particularizada de los fenómenos naturales, su explicación en términos de fuerzas, regularidades y principios inmanentes a aquéllos, no es fácilmente conciliable con el intento de explicar el origen de lo existente, así como del 
lugar y destino del hombre dentro de él, por medio de una causa última, de carácter personal, que por definición sería universal, única, transexperiencial y trascendental.

En realidad, y más allá de lo que Newton y el resto de los científicos ingleses y continentales de su época pudieron haber creído impulsados por sus compromisos metafísico-religiosos, la postulación de una especie de complementariedad entre ciencia y religión no podía sino conducir a una separación final entre ambas. El hecho mismo de que aquella postura no pudiera sostenerse sino a costa de fuertes tensiones internas, así como su final trascendencia en la ciencia posnewtoniana, estarían mostrando que se trataba de un compromiso inestable entre elementos esencialmente irreconciliables. ${ }^{5}$ Pero, claro está, el examen de esta compleja cuestión demandaría un tratamiento especial, y se encuentra más allá de los acotados límites de la presente introducción.

\section{CARTA I}

Al Reverendo Dr. Richard Bentley, en la casa del Obispo de Worcester en Park Street, Westminster.

\section{Señor:}

Cuando escribí mi Tratado acerca de nuestro sistema [del Mundo] ${ }^{6}$ tuve la vista puesta sobre principios tales que pudieran ayudar a los hombres reflexivos en su creencia en una deidad, y nada puede hacerme más feliz que encontrarlo útil para ese propósito. Pero si le he hecho al público algún servicio en este sentido, ello no se debe sino a la diligencia y al paciente pensar.

En cuanto a su primera pregunta, me parece que si la materia de nuestro Sol y sus planetas, y toda la materia del Universo, estuviera uniformemente dispersa (scattered) a través de los cielos, y cada partícula tuviera una gravedad innata hacia todo el resto, y todo el espacio, en el cual toda esta materia

5 Para una versión desarrollada de la interpretación del pensamiento de Newton propuesta en esta introducción, véase mi ensayo titulado: "Ciencia y religión en el pensamiento de Isaac Newton", Revista Universidad de Antioquia, Medellín-Colombia, no. 251, enero-marzo, 1998.

6 Newton alude aquí, por cierto, a su "magnum opus", los Mathematical Principles of Natural Philosophy, cuya primera edición (en latín) vio la luz en 1687. Él denomina "sistema del mundo", a lo que en realidad no era sino una teoría del Sistema Solar. El libro tercero de los Principia (así como su primera redacción publicada posteriormente en forma separada), llevaba por título "De Mundi Systemate", es decir, "El sistema del mundo". Hay ediciones españolas, traducidas por de Eloy Rada García, tanto de los Principios matemáticos de filosofía natural (Alianza, Madrid, 1987, 2 vols.), como de su libro tercero original tituado: El sistema del mundo (Alianza, Madrid, 1992). 
estuvo dispersa, no fuera sino finito; la materia [ubicada] en la parte externa de este espacio tendería por su gravedad hacia toda la materia del interior, y por consecuencia se concentraría en medio del espacio, formando allí una gran masa esférica. Pero si la materia estuviera uniformemente distribuida (disposed) en un espacio infinito, no podría nunca reunirse en una masa, sino que parte de ella se reuniría en una masa y parte de ella en otra, formando así un número infinito de grandes masas, dispersas a grandes distancias unas de otras a través de todo aquel espacio infinito. $Y$ así podrían formarse el Sol y las estrellas fijas, suponiendo que la materia fuera de una naturaleza luminosa (lucid). Pero cómo pudo la materia dividirse en dos clases, y esa parte de ella que es apta para formar un cuerpo brillante pudo caer en una masa y hacer un Sol, y el resto, apta para formar un cuerpo opaco, llegó a unirse (coalesce), no en un gran cuerpo como la materia brillante, sino en muchos pequeños cuerpos; o si el Sol al principio era un cuerpo opaco como los planetas, o los planetas cuerpos luminosos como el Sol, cómo [es que] sólo él se transformó en un cuerpo brillante, mientras todos ellos continuaron [siendo] opacos, o de qué modo todos ellos se transformaron en [cuerpos] opacos, mientras él permaneció inmodificado, yo no lo creo explicable por meras causas naturales, sino que estoy obligado a atribuirlo al propósito e invención de un agente voluntario. ${ }^{7}$

El mismo poder, natural o sobrenatural, que puso al Sol en el centro de los planetas primarios fijos, puso a Saturno en el centro de las órbitas de sus cinco planetas secundarios, y a Júpiter en el centro de sus cuatro planetas secundarios, y a la Tierra en el centro de la órbita de la Luna; y por lo tanto si esta causa hubiera sido ciega, sin artificio ni diseño, el Sol habría sido un cuerpo de la misma clase que Saturno, Júpiter y la Tierra, esto es, sin luz ni calor. No conozco otra razón de por qué hay un [solo] cuerpo en nuestro sistema [solar] capaz de dar luz y calor a todo el resto, sino porque el autor del sistema lo creyó conveniente; y desconozco la razón de por qué no hay más que un cuerpo de esta clase, sino porque uno era suficiente para calentar e iluminar a todo el resto. Porque la hipótesis cartesiana de soles que pierden su luz y luego se transforman en cometas, y de cometas en planetas, ${ }^{8}$ no puede tener cabida en mi sistema, y es claramente errónea; porque es cierto que siempre que aparecen [los cometas] descienden dentro del sistema de

7 Este pasaje reproduce, parcialmente, el lenguaje empleado por Newton en el Scholium general, agregado al libro tercero en la segunda edición (1713) de los Principia, donde se dice que "el más hermoso sistema del Sol, planetas y cometas sólo pudo proceder del consejo y dominio de un ser inteligente y poderoso". Aquí, así como en el resto de los pasajes donde emplea el término 'propósito' (counsel), Newton repite una expresión usada originalmente por Henry More, entre otros, en su escrito de 1652 titulado: An Antidote against Atheism.

${ }^{8}$ Según lo explica Descartes en la parte tercera, párrafo 119, de los Principios de la filosofía. 
nuestros planetas, más bajos que la órbita de Júpiter, y a veces más bajos que las órbitas de Venus y Mercurio, y sin embargo nunca permanecen aquí, sino que siempre regresan desde el Sol con el mismo grado de movimiento con el que se le acercaron.

A su segunda pregunta, contesto que los movimientos que los planetas tienen ahora no pudieron haber brotado sólo de una causa natural, sino que les fueron impresos por un agente inteligente. Pues dado que los cometas descienden dentro de la región de nuestros planetas, y aquí se mueven de diversas maneras, yendo algunas veces en la misma dirección que los planetas, a veces en sentido contrario, y a veces en sentido cruzado, en planos inclinados hacia el plano de la eclíptica, ${ }^{9}$ y en toda clase de ángulos, es claro que no hay una causa natural que pudo determinar a todos los planetas, tanto primarios como secundarios, a moverse en el mismo sentido y en el mismo plano, sin considerable variación: esto debe haber sido el efecto del propósito. Ni hay ninguna causa natural que pudiera dar a los planetas justo aquellos grados de velocidad, en proporción a sus distancias respecto al Sol, y otros cuerpos centrales, que eran requisito para hacerlos moverse en tales órbitas concéntricas en torno a aquellos cuerpos. Si los planetas hubieran sido tan veloces como los cometas, en proporción a sus distancias respecto al Sol (como lo serían si sus movimientos hubieran sido causados por su gravedad, por obra de la cual la materia, en la formación primera de los planetas, pudo haber caído desde las más remotas regiones hacia el Sol), no se moverían en órbitas concéntricas, sino en órbitas tan excéntricas como en las que se mueven los cometas. Si fueran todos los planetas tan veloces como Mercurio, o tan lentos como Saturno o sus satélites; o si fueran sus varias velocidades más o menos grandes de lo que son, como pudieron haberlo sido si hubieran provenido de cualquier otra causa y no de sus gravedades; o si las distancias desde los centros en torno a los cuales se mueven hubieran sido mayores o menores de lo que son, con las mismas velocidades; o si la cantidad de materia en el Sol, o en Saturno, Júpiter y la Tierra en consecuencia su poder gravitacional, hubiera sido mayor o menor de lo que es; los planetas primarios no podrían haber girado en torno al Sol, ni los secundarios en torno a Saturno, Júpiter y la Tierra, en círculos concéntricos como [efectivamente] lo hacen, sino que se habrían movido en hipérbolas o parábolas, o en elipses muy excéntricas. Por lo tanto, hacer este sistema, con todos sus movimientos, requirió una causa que entendiera, y comparara unas con otras, las cantidades de materia en los distintos cuerpos del Sol y

9 La "eclíptica" es el nombre que se usaba en la teoría geocéntrica para indicar el movimiento anual del Sol en torno al cielo, y Copérnico demostró que era puramente aparente. 
los planetas, y los poderes gravitacionales resultantes de ello; las diversas distancias de los planetas primarios respecto al Sol, y de los secundarios respecto a Saturno, Júpiter y la Tierra; y las velocidades con las cuales estos planetas podrían girar en torno a aquellas cantidades de materia en los cuerpos centrales; y comparar y ajustar todas estas cosas conjuntamente, en una variedad tan grande de cuerpos, prueba que aquella causa no es ciega ni fortuita; sino muy bien entrenada en Mecánica y Geometría.

A su tercera pregunta, respondo que puede concebirse que el Sol, al calentar más a aquellos planetas que están más cercanos a él, los hace estar mejor mezclados y más condensados por [obra de] aquella mixtura. Pero cuando considero que nuestra Tierra se calienta mucho más en sus entrañas debajo de la corteza superior por la fermentación subterránea de cuerpos minerales que por el Sol, no veo por qué las partes interiores de Júpiter y Saturno no pudieran estar tan calientes, mezcladas y coaguladas (coagulated) por aquellas fermentaciones como lo está nuestra Tierra; y por lo tanto estas varias densidades deben tener alguna otra causa que las varias distancias de los planetas respecto del Sol. Y me siento confirmado en esta opinión al considerar que los planetas Júpiter y Saturno, en tanto son menos densos (rarer) que el resto, también son bastante mayores [en tamaño], y contienen una cantidad mucho mayor de materia, y tienen muchos satélites girando a su alrededor; características que, seguramente, surgieron no por estar ubicados a una distancia tan grande del Sol, sino que fueron más bien la causa de por qué el Creador los ubicó a una gran distancia, pues por sus poderes gravitacionales alteran muy sensiblemente los movimientos de uno respecto del otro, como lo encuentro [confirmado] por algunas observaciones recientes de Mr. Flamsteed, ${ }^{10}$ y si hubieran sido ubicados mucho más cerca del Sol y uno [respecto] del otro, ellos habrían causado, por los mismos poderes [gravitacionales] una considerable alteración en todo el sistema [solar].

A su cuarta pregunta, respondo que en la hipótesis de los vórtices ${ }^{11}$ la inclinación del eje de la Tierra podría, en mi opinión, ser atribuida a la

10 John Flamsteed (1646-1719), primer "Astrónomo Real" (es decir, el primer director del Observatorio de Greenwich), cuyas observaciones suministraron a Newton parte de los datos astronómicos necesarios para la redacción de los Principia, y en torno a los cuales se suscitó un serio conflicto personal entre ambos.

11 La teoría cartesiana de los vórtices o torbellinos, que aspiraba a explicar el origen y estado presente del Sistema Solar, fue introducida por el filósofo, físico y matemático francés en la tercera parte de su obra Los principios de la filosofía (1644). Su nombre se deriva de los movimientos circulares (tourbillons) que en su modelo mecánico llenarían la totalidad del espacio, los que se suponía arrastrarían consigo a los diferentes planetas, como corchos flotando en el agua. A pesar de su inexactitud matemática, la teoría de los vórtices era fácilmente visualizable, y parecía explicar por qué los planetas giraban en torno al Sol en la misma dirección y aproximadamente en el mismo plano. Pero como observa J.L.E. Dreyer en su obra 
ubicación del vórtice de la Tierra antes de que fuera absorbido por los vórtices vecinos, y de que la Tierra se hubiera transformado de un Sol en un cometa; pero esta inclinación debería decrecer constantemente de acuerdo con el movimiento del vórtice de la Tierra, cuyo eje está mucho menos inclinado hacia la eclíptica, como parece por el movimiento de la Luna llevada allí de un lado a otro. Si el Sol por sus rayos pudiera arrastrar (carry) consigo a los planetas, aun así no veo cómo pudiera él de este modo causar (effect) sus movimientos diarios.

Finalmente, no veo nada extraordinario en la inclinación del eje de la Tierra para probar [la existencia] de una deidad, a menos que Ud. lo forzara (urge) como un artificio [divino] para [crear] el Invierno y el Verano, y para hacer la Tierra habitable cerca de los polos; y que las rotaciones diarias del Sol y los planetas, puesto que difícilmente pudieron haber surgido de una causa puramente mecánica, al estar todas determinadas de la misma manera con movimientos anuales y mensuales, parecen producir (make up) aquella armonia en el sistema, el cual, como lo expliqué más arriba, fue el efecto de elección más bien que del azar. Hay todavía otro argumento en favor de una deidad que considero muy poderoso, pero hasta que los principios sobre los cuales está basado sean mejor recibidos, creo más aconsejable dejarlo dormir. ${ }^{12}$

Yo soy,

su más humilde servidor,

a sus órdenes,

Cambridge,

Diciembre 10, 1692.

Is. NEWTON

clásica: "la teoría cartesiana de los vórtices no daba cuenta de ninguna de las peculiaridades de las órbitas planetarias, y era en realidad pura especulación sin apoyo en hecho alguno" ( $A$ History of Astronomy from Thales to Kepler, Dover Publication, Nueva York, 1953, p. 422). En el libro II de los Principia, Newton dará el golpe mortal a la teoría de los vórtices, al demostrar concluyentemente que los planetas no pueden ser arrastrados por vórtices materiales.

12 En una ponencia presentada ante el XVII Congreso de Historia de la Ciencia, realizado en la Universidad de California, Berkeley, en agosto de 1985, el estudioso norteamericano James Force sostuvo que el argumento que Newton "prefirió dejar dormir" habría sido, en realidad, la demostración del dominio de Dios por medio de las profecías bíblicas correctamente interpretadas. Así lo reporta Richard Olson en su libro Science Deified \& Science Defied. The Historical Significance of Science in Western Culture (University of California Press, Berkeley, 1990, vol. 2, p. 121). 
CARTA II

Para Mr. Bentley, en el Palacio en Worcester.

Señor:

Estoy de acuerdo con Ud. en que si la materia uniformemente dispersa a través del espacio finito, no esférico, llegara a concentrarse en una masa sólida, esta masa afectaría la figura de la totalidad del espacio, siempre que no fuera blanda como el viejo caos, sino tan dura y sólida desde el comienzo que el peso de sus partes protuberantes no pudiera hacerla ceder a su presión. Sin embargo, al ser aflojadas por terremotos las partes de este sólido, las protuberancias podrían a veces hundirse un poco por su peso, y de esta manera la masa, gradualmente, podría aproximarse a una figura esférica.

La razón de por qué la materia uniformemente dispersa a través de un espacio finito se reuniría en medio [de éste], Ud. la concibe al igual que yo; pero que debiera haber una partícula central, tan exactamente ubicada en medio que sería siempre igualmente atraída por todos lados, y que de este modo permaneciera sin movimiento, me parece a mí una suposición tan difícil de aceptar como hacer que la más afilada aguja se pare en su punta sobre un espejo. Porque si el centro matemático de la partícula central no estuviera exactamente en el centro matemático mismo del poder atractivo de toda la masa, la partícula no sería atraída igualmente por todos lados. Y es mucho más difícil suponer que todas las partículas en un espacio infinito pudieran estar tan exactamente equilibradas unas con otras, como para mantenerse inmóviles en un perfecto equilibrio. Porque estimo esto tan difícil como hacer que se paren, exactamente equilibradas sobre sus puntas, no una sola aguja, sino un infinito número de ellas (tantas como hay partículas en un espacio infinito). No obstante, concedo que [esto] es posible, al menos para un poder divino; y si ellas fueran una vez así puestas, estoy de acuerdo con Ud. en que continuarían en esta postura inmóvil por siempre, a menos que fueran puestas de nuevo en movimiento por el mismo poder. Cuando digo, por lo tanto, que la materia uniformemente dispersa a través de todo el espacio se reunirá por su gravedad en una o más grandes masas, me refiero a la materia que no está descansando en un equilibrio exacto.

Pero Ud. argumenta, en el siguiente párrafo de su carta, que cada partícula de materia en un espacio infinito tiene una infinita cantidad de materia por todos lados, y en consecuencia una infinita atracción en todos los sentidos, y [que], por lo tanto, debe reposar en equilibrio, porque todos los infinitos son iguales. Sin embargo, Ud. sospecha un paralogismo en este argumento; y yo pienso que el paralogismo reside en la afirmación (posi- 
- tion) de que todos los infinitos son iguales. La mayoría de la humanidad los considera indefinidos; y en este sentido dicen que todos los infinitos son iguales; aunque hablarian con más verdad si dijeran que no son iguales $\mathrm{ni}$ desiguales, ni tienen ninguna diferencia cierta o proporción entre sí. En este sentido, por lo tanto, no puede extraerse de ellos ninguna conclusión acerca de la igualdad, proporciones o diferencias de las cosas, y aquellos que tratan de hacerlo caen usualmente en paralogismos. De modo que cuando los hombres argumentan en contra de la infinita divisibilidad de la magnitud, diciendo que si una pulgada puede ser dividida en un infinito número de partes, la suma de tales partes será una pulgada; y si un pie puede ser dividido en un infinito número de partes, la suma de dichas partes debe ser un pie, y en consecuencia, dado que todos los infinitos son iguales, aquellas sumas deben ser iguales, esto es, una pulgada debe ser igual a un pie.

La falsedad de la conclusión muestra un error en las premisas, y el error reside en la afirmación (position) de que todos los infinitos son iguales. Hay, por lo tanto, otra manera empleada por los matemáticos, y es aquélla por medio de la cual se determina que los infinitos guardan ciertas diferencias o proporciones unos respecto de otros, en ciertas restricciones y limitaciones definidas. Así lo considera el Dr. Wallis ${ }^{13}$ en su Arithmetica infinitorum, en la que por las diferentes proporciones de las sumas infinitas él infiere las diferentes proporciones de las magnitudes infinitas; manera de argumentar generalmente aceptada por los matemáticos, y que sin embargo no sería buena si todos los infinitos fueran iguales. De acuerdo con el mismo modo de considerar los infinitos, un matemático le diría que aunque haya un número infinito de pequeñas partes infinitas en una pulgada, sin embargo habrá doce veces aquel número de tales partes en un pie, esto es, el número infinito de aquellas partes en un pie no es igual, sino doce veces más grande que el infinito número de ellas en una pulgada. Y así un matemático le dirá que si un cuerpo se mantuvo en equilibrio entre dos fuerzas atractivas infinitas iguales y contrarias; y si a cualquiera de estas fuerzas Ud. agrega una nueva fuerza atractiva finita, esa nueva fuerza, no importa cuán pequeña sea, romperá su equilibrio, y pondrá al cuerpo en el mismo movimiento en el cual lo hubiera puesto si aquéllas hubieran sido dos fuerzas iguales y contrarias, pero finitas, o incluso ninguna; de modo que en este caso los dos infinitos iguales, por la agregación de un finito a cualquiera de ellos,

13 John Wallis (1616-1703), uno de los fundadores de la Royal Society, y el más grande matemático inglés anterior a Newton, cuyos trabajos sobre análisis infinitesimal contribuyeron al descubrimiento del Cálculo Diferencial e Integral por Newton y Leibniz. En 1649 Wallis fue nombrado "Savilian Professor" de Geometría en la Universidad de Oxford. Junto con la Arithmetica infinitorum (1655), referida en esta carta, escribió un Treatise of Algebra, Both Historical and Practical, que fue publicado en 1685. Fuente: A History of Mathematics, de Carl B. Boyer (John Wiley \& Sons, Nueva York, 1991, pp. 379, 380 y 382). 
devienen desiguales en nuestra manera de calcular; y después de estos pasos (ways) debemos estimar si de la consideración de los infinitos podríamos extraer siempre conclusiones verdaderas.

A la última parte de su carta, respondo, primero, que si la Tierra (sin la Luna) fuera colocada en algún lugar con su centro en la "Orbis Magnus", ${ }^{14}$ y se mantuviera fija allí sin ninguna gravitación ni proyección, y allí en el acto le fuera insuflada, tanto una energía gravitante hacia el Sol, como un impulso transversal de una cantidad precisa que la moviera directamente en una tangente con la "Orbis Magnus"; los componentes de esta atracción y proyección causarían, de acuerdo con mi idea, una revolución circular de la tierra en torno al Sol. Pero el impulso transversal debe ser de una cantidad precisa; porque si fuera demasiado grande o demasiado pequeña, haría que la Tierra se moviera en cualquiera otra línea. En segundo lugar, yo no conozco ningún poder en la naturaleza que pudiera causar este movimiento transversal sin el brazo divino. Blondel nos dice en alguna parte de su Libro de las bombas, ${ }^{15}$ que Platón afirma que el movimiento de los planetas es tal que [pareciera] como si todos ellos hubieran sido creados por Dios en alguna región muy remota de nuestro Sistema [Solar], y dejados caer desde alli hacia el Sol, y que tan pronto como arribaron a sus diferentes órbitas sus movimientos de caída se transformaron en un movimiento transversal. $Y$ esto es verdad, suponiendo que el poder gravitacional del Sol, en aquel momento en que todos los planetas arribaron a sus distintas órbitas, fuera el doble; pero entonces el poder divino es requerido aquí para dos cosas, esto es, para transformar los movimientos de descenso de los planetas en caída en un movimiento lateral, y para, al mismo tiempo, duplicar el poder atractivo del Sol. Así, entonces, la gravedad pudo haber puesto los planetas en movimiento, pero sin el poder divino no podría nunca haberlos puesto en un movimiento circulatorio tal como el que tienen en torno al Sol; y por lo tanto, por ésta, así como por otras razones, estoy compelido a atribuir la construcción (frame) de este sistema a un agente inteligente.

14 "Orbis Magnus", término acuñado por Copérnico para denotar la revolución u órbita anual de la Tierra en torno al Sol, que pasaría a formar parte del vocabulario astronómico de los siglos xv y xvi. Como lo indica Kepler en su Epítome de astronomía copernicana, lo de magnus no se refiere a su tamaño, "puesto que las órbitas circulares de los planetas superiores son mucho más grandes, sino en razón de su extraordinaria utilidad en salvar los movimientos aparentes, no sólo del Sol, sino también de los planetas primarios" (citado por Edward Rosen en su Introducción a Three Copernican Treatises, Dover Publications, Nueva York, 1959, p. 17).

15 Newton alude aquí a $L^{\prime}$ art de jetter les bombes, de Monsieur Blondel (Maréchal de Camp aux Armées du Roy, Amsterdam, 1683, 3a. parte, libro primero, "Doctrine de Galilée sur le mouvement", cap. VII, p. 166). Referido por Alexandre Koyré en Newton, Galileo and Plato, Newtonian Studies (University of Chicago Press, Chicago, 1968, p. 206). 
Usted habla a veces de la gravedad como esencial e inherente a la materia. Le ruego no me atribuya a mí tal noción; ${ }^{16}$ porque yo no pretendo saber cuál es la causa de la gravedad, y por tanto tomaría más tiempo considerarla. ${ }^{17}$

Me temo que lo que he dicho de los infinitos le parecerá a Ud. oscuro; pero es suficiente que Ud. comprenda que los infinitos, cuando se los considera absolutamente sin ninguna restricción ni limitación, no son iguales ni desiguales, ni guardan proporciones ciertas (certain) entre sí, y por lo tanto, el principio de que todos los infinitos son iguales es precario.

\author{
Yo soy, \\ su más humilde servidor, \\ Trinity College, \\ Enero 17, 1692-1693. \\ Is. NEWTON
}

\title{
CARTA III
}

Para Mr. Bentley, en el Palacio en Worcester.

Señor:

Puesto que Ud. desea rapidez, responderé su carta con tanta brevedad como pueda. Estoy de acuerdo con Ud. en las seis posiciones que establece al comienzo de su carta. Su suposición de que la "Orbis Magnus" es 7000 diámetros del ancho de la tierra, implica que la paralaje horizontal del Sol es medio minuto [de arco]. Flamsteed y Cassini ${ }^{18}$ han observado, recientemente, que es de alrededor de 10", y así la "Orbis Magnus" debe ser 21.000,

16 En la única carta de Bentley a Newton que se conserva, la tercera, aquél se disculpa señalando que él no afirma que la gravedad sea una propiedad innata de la materia, que no asigna tal doctrina al físico, astrónomo y matemático inglés, y que si usó tal expresión fue únicamente por motivos de brevedad. Así lo reporta Koyré en en el capítulo IV, p. 208, de sus Newtonian Studies, recién referidos.

17 En el último párrafo del Scholium general a la segunda edición de los Principia, Newton había declarado: "Pero hasta ahora no he podido descubrir la causa de aquellas propiedades de la gravedad a partir de los fenómenos. [...] Y para nosotros es suficiente que la gravedad exista realmente, y que actúe de acuerdo con las leyes que hemos explicado, y abundantemente sirven para explicar todos los movimientos de los cuerpos celestes, y de nuestro mar" (Mathematical Principles of Natural Philosophy, Motte y Cajori, Encyclopaedia Britannica, Chicago, 1978, vol. 34, pp. 371-372).

18 Newton se refiere, muy probablemente, al astrónomo Gian Domenico Cassini (16251712), acerca de quien escribe Charles Singer lo siguiente: "comenzó siendo un ingeniero al servicio papal. Estableció una reputación astronómica por sus escritos acerca de los cometas (1652), y por sus observaciones sobre los periodos de rotación de Marte, Júpiter y Venus (1665-1667). Fue llamado a París por Luis XIV en 1669, llegando a ser la figura más influ- 
o en un número más redondo, 20.000 diámetros del ancho (wide) de la Tierra. Cualquiera de los dos cómputos, creo yo, está bien, y no creo que valga la pena alterar sus números.

En la parte siguiente de su carta Ud. establece otras cuatro posiciones, basadas sobre una primera fija. La primera de estas cuatro parece muy evidente, suponiendo que Ud. considera la atracción de modo tan general que se entienda por ella cualquier fuerza por la cual cuerpos distantes tratan de acercarse sin impulso mecánico. La segunda no parece tan clara; pues puede decirse que pudieron haber [existido] otros sistemas planetarios (systems of worlds) antes de los actuales, y otros antes que aquellos, y así sucesivamente para toda la eternidad pasada, y en consecuencia que la gravedad puede ser coeterna con la materia, y tener el mismo efecto por toda la eternidad que como en el presente, al menos que Ud. haya probado en alguna parte que los sistemas viejos no pueden convertirse gradualmente en nuevos; o que este sistema no tenga su origen en la materia emanada de sistemas anteriores en descomposición, sino en un caos de materia uniformemente dispersa a través de todo el espacio; pues algo de esta clase, creo, dijo Ud. que era el tema de su quinto sermón; y el crecimiento de nuevos sistemas a partir de los antiguos, sin la mediación del poder divino, me parece a mí [algo] manifiestamente absurdo.

La última cláusula de su segunda posición me gusta mucho. Es inconcebible que la materia bruta inanimada pudiera, sin la mediación de algo más, que no fuera material, operar sobre y afectar a otra materia sin contacto mutuo, como debiera ser si la gravitación en el sentido de Epicuro es esencial e inherente a ella. $Y$ esta es una razón de por qué desearía que Ud. no me atribuyera a mí la gravedad innata. Que la gravedad debiera ser innata, inherente y esencial a la materia, de modo que un cuerpo pudiera actuar sobre otro a la distancia a través de un Vacío, sin la mediación de ninguna otra cosa, por y a través del cual sus acciones y fuerzas pudieran ser transmitidas de uno a otro, es para mí un absurdo tan grande que creo que ningún hombre que tenga en materias filosóficas una facultad competente de pensamiento puede caer jamás en él. La gravedad debe ser causada por un agente que actúa constantemente de acuerdo con ciertas leyes; pero

yente en el observatorio real. Bajo sus auspicios se mostró que la Tierra era achatada en los polos, un descubrimiento que tuvo importantes implicaciones astronómicas. También bajo su dirección, fue medida la paralaje de Marte. Esto condujo a una estimación de la distancia de la Tierra respecto del Sol, que aunque fue la mejor hasta esa fecha, estaba equivocada en alrededor de $7 \%$. Cassini era un hombre convencionalmente piadoso y, notable en aquella fecha, un anticopernicano. Fue sucedido en el Observatorio de París por tres generaciones de descendientes" (Charles Singer, A Short History of Scientific Ideas, Oxford University Press, Nueva York y Londres, 1959, p. 304). 
determinar si este agente es material o inmaterial lo he dejado a la consideración de mis lectores.

Su cuarta aserción, que el mundo no pudo ser formado sólo por gravedad innata, Ud. la confirma por tres argumentos. Pero en su primer argumento parece cometer una Petitio Principii; porque mientras muchos filósofos antiguos y otros, así como teístas y ateos, han todos aceptado que pudiera haber mundos y porciones de materia innumerable o infinita, Ud. niega esto, considerándolo tan absurdo como la idea de que pudiera positivamente haber una infinita suma aritmética de números, lo que es una contradicción in Terminis; pero Ud. no prueba que sea absurdo. Tampoco prueba Ud. que lo que los hombres quieren decir por una suma infinita o número infinito es una contradicción en la naturaleza; porque una contradicción in Terminis no implica más que una impropiedad del lenguaje. Lo que los hombres entienden por frases impropias y contradictorias pueden ser a veces reales en la naturaleza [y] sin la menor contradicción: un tintero de plata, una linterna de papel, una piedra de afilar, son frases (phrases) absurdas, ${ }^{19}$ sin embargo las cosas significadas con ellas se encuentran realmente en la naturaleza. Si algún hombre dijera que un número y una suma, propiamente hablando, es aquello que puede ser enumerado y sumado, pero que las cosas infinitas no tienen número (numberless), o como decimos usualmente, son innumerables y sin suma, o insumables, y que por lo tanto no deberían ser llamadas un número o una suma, aquel que así lo hace hablará con suficiente propiedad y su argumento en contra de él perderá, me temo, su fuerza. $\mathrm{Y}$, sin embargo, si algún hombre tomara las palabras "número" y "suma" en un sentido más amplio, a manera de entender por medio de ellas cosas que en la forma propia de hablar son innumerables e insumables (como Ud. parece hacerlo cuando acepta un infinito número de puntos en una línea) yo podría fácilmente permitirle a él el uso de las frases contradictorias de número innumerable, o de suma insumable, sin inferir a partir de ello ningún absurdo en lo que quiere decir con tales expresiones. Sin embargo, si por este o cualquier otro argumento, Ud. ha probado la infinitud del Universo, se sigue que toda materia caerá desde los lados y se juntará en el centro [del espacio]. Sin embargo, la materia al caer podría concretarse en muchas masas redondas, como los cuerpos de los planetas, y éstos al atraerse unos a otros podrían adquirir una oblicuidad de descenso, por medio de la cual podrían caer, no sobre el gran cuerpo central, sino a su lado, y dar un rodeo

19 Las expresiones que Newton considera aquí como absurdas y contradictorias no se revelan como tales sino al ser traducidas en forma literal al español. Así, un tintero de plata (silver inkhorn) se transforma en un "cuerno de tinta de plata"; una linterna de papel (paper lanthorn) deviene una "lámpara de cuerno de papel", mientras que una piedra de afilar (iron whetstone) se convierte en una "piedra mojada de hierro". 
en torno a él, y entonces volver a ascender por los mismos pasos y grados de movimiento y velocidad con los cuales descendieron antes, de modo muy semejante a como los cometas giran en torno al Sol; pero no podrían adquirir nunca por la sola gravedad un movimiento circular en órbitas concéntricas alrededor del Sol.

$\mathrm{Y}$ aunque toda la materia fuera dividida al comienzo en varios sistemas, y cada sistema fuera constituido como el nuestro por un poder divino, de todos modos los sistemas externos podrían descender hacia la masa más central; de modo que este orden (frame) de cosas no podría subsistir siempre sin un poder divino que lo conserve, lo que es el segundo argumento; y [en cuanto] a su tercero yo asiento completamente.

En cuanto al pasaje de Platón, ${ }^{20}$ no hay un lugar común desde el cual todos los planetas fueron dejados caer, y descendiendo con gravedades uniformes e iguales (como supuso Galileo), ${ }^{21}$ al llegar a sus distintas órbitas adquirieran las distintas velocidades con las que hoy giran. Si suponemos que la gravedad de todos los planetas hacia el Sol es de tal cantidad (quantity) como la que realmente es, y que los movimientos de los planetas fueron convertidos en ascendentes (turned upwards), cada planeta subirá al doble de su altura respecto al Sol. Saturno ascenderá hasta encontrarse al doble de la altura respecto al Sol en que está en el presente, y no más alto; Júpiter ascenderá tan alto como está en el presente, esto es, un poco por sobre la órbita de Saturno; Mercurio ascenderá al doble de su altura presente, esto es, hasta la órbita de Venus; y asimismo el resto [de los planetas]; y entonces

20 Esta hipótesis, que según ha demostrado concluyentemente Koyré no se encuentra en la obra de Platón, es examinada por Galileo, tanto en la Primera jornada de su Diálogo sobre los dos máximos sistemas (1632), como en el Cuarto día de sus Discursos y Demostraciones matemáticas sobre dos nuevas ciencias (1638). En la primera de estas obras la hipótesis es introducida por Salviati del siguiente modo: "A no ser que quisiéramos decir con Platón que también los cuerpos del mundo [es decir, los planetas], después de haber sido construidos y totalmente acabados, durante algún tiempo fueron movidos por su autor con movimiento rectilíneo, pero que después de haber llegado a determinados lugares, fueron reorientados uno a uno en círculo pasando del movimiento rectilíneo al circular, en los que todavia se mantienen y siempre se conservan. Pensamiento éste sublime y digno de Platón. . " (Galileo Galilei, Diálogo sobre los dos máximos sistemas del mundo ptolemaico y copernicano, trad. Antonio Beltrán Marí, Alianza Editorial, Madrid, 1994, p. 19).

21 La pregunta que dio origen a la respuesta de Newton que ocupará el resto de esta carta, fue formulada textualmente por Bentley en su tercera carta a aquél (del 18 de febrero de 1693), en los siguientes términos: "Ahora, por lo tanto, supóngase que los planetas son formados en algunas regiones más altas [del espacio], descendiendo primero hacia el Sol donde adquirirán sus velocidades; pero entonces ellos habrían continuado sus descensos hacia el Sol, a menos que un poder divino les diera un movimiento transversal opuesto al vasto ímpetu con el que aquellos grandes cuerpos debieron caer; de manera que de todos modos hay necesidad de introducir un Dios" (reproducido por Alexandre Koyré en la nota 2 de la p. 208 de sus Newtonian Studies antes citados). 
al caer nuevamente desde los lugares a los cuales ascendieron, arribarán de nuevo a sus diferentes órbitas con las mismas velocidades que tenían al principio, y con las cuales ahora giran.

Pero si tan pronto como los movimientos giratorios de los planetas son transformados en ascendentes el poder gravitacional del Sol, por el cual su ascenso es perpetuamente retardado, es disminuido a la mitad, los planetas ascenderán perpetuamente, y todos los [que estén] a iguales distancias desde el Sol se moverán con igual rapidez. Cuando Mercurio llegue a la órbita de Venus, será tan rápido como éste; y cuando él y Venus lleguen a la órbita de la Tierra, serán tan rápidos como ésta, y así igualmente el resto. Si todos ellos comienzan a ascender a un mismo tiempo, y lo hacen en la misma línea, se irán acercando cada vez más unos a otros, y sus movimientos se aproximarán constantemente a una igualdad, y a la larga se harán más lentos que ningún movimiento asignable. Supóngase, por lo tanto, que ascendieran hasta casi tocarse y sus movimientos fueran insignificantemente pequeños, y que todos volvieran a ser otra vez devueltos (turned back) [a sus órbitas] al mismo tiempo; o lo que viene a ser casi la misma cosa, que fueran privados sólo de sus movimientos, y dejados caer en ese momento; todos arribarían al mismo tiempo a sus distintas órbitas, cada cual con la velocidad que tenía al principio; y si sus movimientos fueran entonces transformados en transversales (turned sideways), y al mismo tiempo se duplicara el poder gravitacional del Sol [de tal modo] que pudiera ser lo suficientemente poderoso como para retenerlos en sus órbitas, los planetas girarían en ellas como antes de su ascenso. Pero si el poder gravitacional del Sol no fuera duplicado, los planetas se saldrían de sus órbitas, en líneas parabólicas, y en la dirección de los más altos cielos. Estas cosas se siguen de mis Princ. Math, Lib. 1, Prop. 33, 34, 36, 37.

Su más,

humilde servidor,

a sus órdenes,

Cambridge,

Febrero 25, 1692.

Is. NEWTON 
CARTA IV

A Mr. Bentley, en el Palacio en Worcester.

\section{Señor:}

La hipótesis de derivar la estructura del mundo por [medio] de principios mecánicos de la materia uniformemente distribuida a través de los cielos, por ser inconsistente con mi sistema, la he considerado muy poco antes de que sus cartas me hicieran poner atención sobre ella, y por lo tanto lo molesto con una línea o dos más al respecto, si es que esto no llega demasiado tarde para su uso.

En mi [carta] anterior yo le manifesté que las rotaciones diarias (diurnal) de los planetas no podían derivarse de la gravedad, sino que requerían haber sido impuestas por un brazo divino. $\mathrm{Y}$ aunque la gravedad pudiera dar a los planetas un movimiento de descenso hacia el Sol, ya sea directamente o con alguna pequeña oblicuidad, los movimientos transversales por los cuales giran en sus diferentes órbitas requirieron el brazo divino para que se los impusiera de acuerdo con las tangentes de sus órbitas. Yo agregaría ahora que la hipótesis de que la materia estaba al principio uniformemente dispersa a través de los cielos, es, en mi opinión, inconsistente con la hipótesis de la gravedad innata sin un poder sobrenatural que las reconcilie, y por tanto implica una deidad. Pues si hay gravedad innata sería imposible para la materia de la Tierra y de todos los planetas y estrellas escaparse de ella y llegar a estar uniformemente repartida a través de todo el cielo sin un poder sobrenatural; y ciertamente, aquello que no puede nunca llegar a ser en el futuro sin un poder sobrenatural, no podría haberlo sido nunca en el pasado sin el mismo poder.

Usted preguntaba si acaso la materia uniformente dispersa a través de un espacio finito, de otra figura que la esférica, al caer hacia un cuerpo central no haría a aquel cuerpo ser de la misma figura que la totalidad del espacio, y yo le respondí que sí. Pero en mi respuesta debe suponerse que la materia desciende directamente hacia aquel cuerpo, y que ese cuerpo no tiene rotación diaria.

Esto, señor, es todo lo que yo agregaría a mis cartas anteriores.

Soy,

su más modesto,

siervo,

Cambridge,

Feb. 11, 1693.

Is. NewTON

Finis

Introducción, traducción y notas de Hermes H. BeniteZ 